\title{
Probiotics improved the effectiveness and safety of the quadruple Helicobacter pylori eradication therapy.
}

\author{
Lili Jiang*, Weihua Zhu \\ Department of Gastroenterology, General Hospital of Xinjiang Military Region, Urumqi, PR China
}

\begin{abstract}
Background: Helicobacter pylori $(\mathrm{Hp})$ infection was prevalent all over the world, but the Hp eradication rate remained low. This randomized controlled double blinded study aimed investigated the effectiveness and safety of probiotics combined with the standard regimen in eradicating $\mathrm{Hp}$ infection.

Methods: Consecutive adult patients who had C14 breath test positivity (>100) and endoscopically diagnosed as chronic active gastritis or gastric ulcer were enrolled and randomized into control group $(n=111)$ and experimental group $(n=111)$. Demographic and clinical characteristics were recoded. The Hp eradication rate and adverse events were compared between two groups.

Results: The demographic and clinical characteristics were comparable between the control and experimental group (all $P$ values $>0.05$ ). The effectiveness rate of control group was $84.7 \%$, which was obviously lower than that of the experimental group (84.7\% vs. 94.6\%, $P=0.041)$. The total incidence of the adverse events in control group was also statistically higher than that in experimental group $(P=0.023)$. There were no statistically significant differences on the rate of taste disorders and anorexia between the two groups $(\mathrm{P}>\mathbf{0 . 0 5})$. The incidences of abdominal distension, diarrhoea and constipation in control group were greatly higher than those in experimental group (all $P$ values $<0.05$ ).

Conclusion: These findings proved that the probiotics can improve the effectiveness and safety of the standard Hp eradication regimen.
\end{abstract}

Keywords: Helicobacter pylori, Probiotics, Treatment, Effectiveness, Safety.

Accepted on March 23, 2018

\section{Introduction}

Helicobacter pylori $(\mathrm{Hp})$ infection has been proved to be an important etiological factor for multiple gastric diseases including chronic gastritis, peptic ulcer, gastric mucosal associated lymphoma and gastric cancer [1,2]. Although great progress has been made on the diagnosis and treatment for $\mathrm{Hp}$ infection, its prevalence remains high both at home and abroad $[3,4]$. In our country, it was estimated that around $60 \%$ of the population has $\mathrm{Hp}$ infection [5]. Most of the patients with $\mathrm{Hp}$ infection are asymptomatic, and some patients only have some nonspecific symptoms like abdominal distention and dyspepsia, which can be easily neglected. However, Hp infection can increase the risk of gastric and esophageal cancer [6]. Thus, it is necessary to guarantee the eradication of $\mathrm{Hp}$ infection.

At present, the eradication rate of $\mathrm{Hp}$ infection is on the decrease. The main reason may be the development of the drug resistance. Previous studies showed that the combination of probiotics and standard $\mathrm{Hp}$ eradication can improve the eradication of $\mathrm{Hp}$, while these studies were mainly based on an observational method [7,8]. Our current study was designed to investigate the effects of probiotics on the $\mathrm{Hp}$ eradication therapy, which may provide evidence for optimizing the regimen for eradicating $\mathrm{Hp}$ infection.

\section{Patients and Methods}

\section{Patients}

Consecutive adult patients who had $\mathrm{C} 14$ breath test positivity $(>100)$ and endoscopically diagnosed as chronic active gastritis or gastric ulcer in our outpatient department of gastroenterology from May 1, 2017 to October 1, 2017 were enrolled. Those patients who were unwilling to participate in this study, pregnant or lactating, or had severe cardiac, hepatic, renal or malignant diseases, or previously took anti-Hp drugs including antibacterial drugs, bismuth agents and proton pump inhibitors were excluded.

The demographic (age, gender, etc.) and clinical characteristics (main symptoms, etc.) were recorded. All the patients gave their written informed consents. This study was approved by the Ethic Committee of Xinjiang military area general hospital and the protocol was in accordance with the Declaration of Helsinki. 


\section{Study design}

This study was designed as a randomized controlled double blinded clinical trial and the flow chart was shown in Figure 1. A total of 232 patients were enrolled and 10 patients were excluded because they were unwilling to participate. The eligible patients were randomly divided into control group $(n=111)$ and experimental group $(n=111)$ using random number method. The patients and the doctors were both blinded to the grouping information. The control group was treated by a quadruple Helicobacter pylori eradication regimen of colloidal bismuth pectin $(0.2 \mathrm{~g}$ bid $)$, lansoprazole $(15 \mathrm{mg}$ bid $)$, amoxicillin (1.0 g bid) and clarithromycin $(0.5 \mathrm{~g}$ bid) for 2 weeks, while the experimental group was treated by a quadruple Helicobacter pylori eradication regimen of colloidal bismuth pectin $(0.2 \mathrm{~g}$ bid $)$, lansoprazole (15 $\mathrm{mg}$ bid $)$, amoxicillin ( $1.0 \mathrm{~g}$ bid) and clarithromycin $(0.5 \mathrm{~g}$ bid) plus live combined bifidobacterium ( 2 capsules tid) for 2 weeks. All the patients in the two groups were equally monitored during the study.

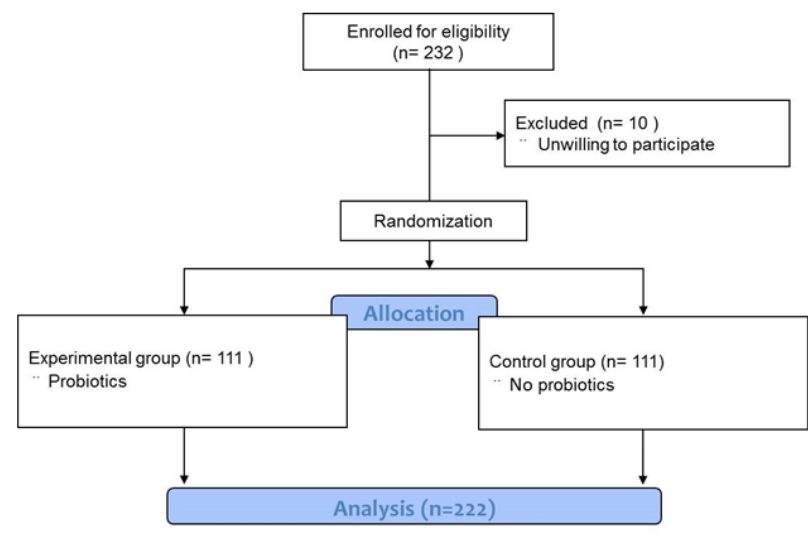

Figure 1. Flow chart of the study.

\section{Outcomes}

The main outcome was the effectiveness rate of $\mathrm{Hp}$ eradication, which was defined as the negativity of $\mathrm{C} 14$ breath test at 1 month after the eradication.

The second outcome was the rate of adverse event including taste disorders, anorexia, abdominal distension, diarrhoea and constipation during the study period.

\section{Statistical analysis}

All the statistical analysis was performed using SPSS 17.0 software (SPSS Inc., Chicago, IL, USA).

The continuous and categorical data were presented as mean (range) and percentage (\%), and the differences between the two groups were compared by independent student t-test and chi-square test if applicable, respectively.

A two tailed $\mathrm{P}$ value less than 0.05 was considered to be statistically significant.

\section{Results}

\section{Demographic and clinical characteristics}

No significantly statistical differences were found on the demographic and clinical characteristics between the control and experimental group (all $\mathrm{P}$ values $>0.05$ ) (Table 1). The mean age of the patients in control and experimental group was 34.8 and 35.2 y old $(\mathrm{P}=0.221)$. There were 50 female and 61 male patients in control group, and there were 48 female and 63 male patients in experimental group $(\mathrm{P}=0.309)$. In control group, 78 patients had heart burn, 45 patients had abdominal pain, 101 patients had dyspepsia and 88 patients had abdominal distention. In experimental group, 83 patients had heart burn, 49 patients had abdominal pain, 99 patients had dyspepsia and 89 patients had abdominal distention, which was comparable with control group (all $\mathrm{P}$ values $>0.05$ ).

Table 1. Demographic and clinical characteristics.

\begin{tabular}{|c|c|c|c|}
\hline & $\begin{array}{l}\text { Control group } \\
(n=111)\end{array}$ & $\begin{array}{l}\text { Experimental } \\
\text { group }(n=111)\end{array}$ & $P$ value \\
\hline Age, years, mean (range) & $34.8(20-54)$ & $35.2(19-58)$ & 0.221 \\
\hline \multicolumn{4}{|l|}{ Gender, n (\%) } \\
\hline Female & $50(45.0)$ & $48(43.2)$ & \multirow[t]{2}{*}{0.309} \\
\hline Male & $61(55.0)$ & $63(56.8)$ & \\
\hline \multicolumn{4}{|l|}{ Clinical manifestation, $\mathrm{n}(\%)$} \\
\hline Heart burn & $78(70.3)$ & $83(74.8)$ & 0.434 \\
\hline Abdominal pain & $45(40.5)$ & $49(44.1)$ & 0.412 \\
\hline Dyspepsia & $101(91.0)$ & $99(89.2)$ & 0.711 \\
\hline Abdominal distention & $88(79.3)$ & $89(80.2)$ & 0.506 \\
\hline
\end{tabular}

\section{Effectiveness and safety evaluation}

The effectiveness rate of control group was $84.7 \%$, which was obviously lower than that of the experimental group $(84.7 \% \mathrm{vs}$. $94.6 \%, \mathrm{P}=0.041$ ) (Table 2). All the adverse events in the study period were recorded. There were no statistically significant differences on the rate of taste disorders and anorexia between the two groups. The incidences of abdominal distention, diarrhoea and constipation in control group were greatly higher than those in experimental group (all $\mathrm{P}$ values $<0.05$ ). The total incidence of the adverse events in control group was also statistically higher than that in experimental group $(\mathrm{P}=0.023)$. No severe adverse events occurred and all the adverse events disappeared at 1-month follow-up after the regimen.

Table 2. Effectiveness and safety evaluations.

\begin{tabular}{lllll}
\hline & $\begin{array}{l}\text { Control } \\
(\mathbf{n = 1 1 1 )}\end{array}$ & group & $\begin{array}{l}\text { Experimental } \\
\text { group (n=111) }\end{array}$ & P value \\
\hline Effectiveness rate, \% & $84.7(94 / 111)$ & $94.6(105 / 111)$ & 0.041 \\
\hline Adverse events, $\mathrm{n}(\%)$ & & & \\
\hline Taste disorders & $1(0.9)$ & $1(0.9)$ & 0.308 \\
\hline
\end{tabular}




\begin{tabular}{llll}
\hline Anorexia & $1(0.9)$ & $1(0.9)$ & 0.202 \\
\hline Abdominal distension & $4(3.6)$ & $2(1.8)$ & 0.035 \\
\hline Diarrhoea & $18(16.2)$ & $2(1.8)$ & 0.009 \\
\hline Constipation & $2(1.8)$ & $1(0.9)$ & 0.049 \\
\hline Total & $26(23.4)$ & $7(6.3)$ & 0.023 \\
\hline
\end{tabular}

Future large scale multi-center investigations are also needed to validate the role of probiotics in the eradication of $\mathrm{Hp}$.

\section{References}

1. Varga MG, Wang T, Cai H. Helicobacter pylori blood biomarkers and gastric cancer survival in china. Cancer epidemiology, biomarkers \& prevention. Am Association Cancer Res 2018; 27: 342-344.

\section{Discussion}

Hp infection has been important risk factors for gastritis, peptic ulcer and gastric cancer. The overall prevalence of Hp infection remained high and it can reach as high as $60 \%$ in China. In recent years, with the application of antibiotics in the eradication of $\mathrm{Hp}$, drug resistance and micro-environmental changes could cause the ineffectiveness of the regimen and the increase of the adverse events [9]. Probiotics can benefit the human healthy by regulating the immune function and medicating the normal functional activities, especially in the gastrointestinal tract [10]. The potential molecular mechanism may be involved in the anti-oxidative and anti-inflammatory activities which are able to protect the gastric mucosa, suppress the inflammatory and immune response and rebalance the micro ecosystem after Hp infection [11]. Seen from this, the combination of probiotics and the standard $\mathrm{Hp}$ eradication regimen may improve the $\mathrm{Hp}$ eradication rate [12].

In this study, our findings proved that probiotics can significantly improve the effectiveness of the standard regimen and decrease the incidence of adverse events. The effectiveness rate of experimental group was obviously higher than that of the control group $(94.6 \%$ vs. $84.7 \%, \mathrm{P}=0.041)$. The total incidence of the adverse events in experimental group was obviously decreased compared with that in control group $(\mathrm{P}=0.023)$. Although the incidences of taste disorders and anorexia were comparable between the two groups, the incidences of abdominal distention, diarrhoea and constipation in control group were greatly decreased in experimental group (all $\mathrm{P}$ values $<0.05$ ). Taste disorders and anorexia were mainly associated with the regimen itself, while abdominal distention, diarrhoea and constipation were closely correlated with the intestinal micro ecosystem, which can be regulated by the administration of probiotics. Our results were consistent with previous studies [7,13,14]. In vitro experiment showed that the probiotics can inhibit and kill $\mathrm{Hp}$ and prevent the release of interleukin-8 [15]. In addition, the probiotics can alleviate the gastric mucosal inflammation caused by $\mathrm{Hp}$ infection and decrease the incidence of antibiotics associated diarrhoea [16].

There were still limitations in this study. First, all the patients were from one single center, and those who had ever received such Hp eradication regimen were excluded. Second, there may be other factors influencing the therapeutic efficacy of the $\mathrm{Hp}$ eradication like dietary factors and the like, which have not been evaluated.

Taken together, our findings supported that the probiotics can be introduced to the current $\mathrm{Hp}$ eradication standard regimen, which improved both the therapeutic effectiveness and safety.

2. Conteduca V, Sansonno D, Lauletta G, Russi S, Ingravallo G, Dammacco F. H. pylori infection and gastric cancer: state of the art (review). Int J Oncol 2013; 42: 5-18.

3. Meng W, Bai B, Sheng L. Role of Helicobacter pylori in gastric cancer: advances and controversies. Discovery Med 2015; 20: 285-293.

4. Feng L, Wen MY, Zhu YJ, Men RT, Yang L. Sequential therapy or standard triple therapy for helicobacter pylori infection: An updated systematic review. Am J Therap 2016; 23: 880-893.

5. Zamani M, Ebrahimtabar F, Zamani V. Systematic review with meta-analysis: the worldwide prevalence of Helicobacter pylori infection. Alimentary Pharmacol Therap 2018.

6. Polyzos SA, Zeglinas C, Artemaki F. Helicobacter pylori infection and esophageal adenocarcinoma: a review and a personal view. Ann Gastroenterol 2018; 31: 8-13.

7. Iakovenko EP, Grigor'ev P, Iakovenko AV. Effects of probiotic bifiform on efficacy of Helicobacter pylori infection treatment. Terapevticheskii Arkhiv 2006; 78: 21-26.

8. Armuzzi A, Cremonini F, Ojetti V. Effect of Lactobacillus GG supplementation on antibiotic-associated gastrointestinal side effects during Helicobacter pylori eradication therapy: a pilot study. Digestion 2001; 63: 1-7.

9. Mascellino MT, Porowska B, De Angelis M, Oliva A. Antibiotic susceptibility, heteroresistance, and updated treatment strategies in Helicobacter pylori infection. Drug Design, Development and Therapy 2017; 11: 2209-2220.

10. Barko PC, McMichael MA, Swanson KS, Williams DA. The gastrointestinal microbiome: A review. J Veterinary Internal Med 2018; 32: 9-25.

11. Yang L, Tian ZB, Yu YNl. Saccharomyces boulardii administration can inhibit the formation of gastric lymphoid follicles induced by Helicobacter suis infection. Pathogens Dis 2017; 75.

12. Goderska K, Agudo Pena S, Alarcon T. Helicobacter pylori treatment: antibiotics or probiotics. Applied Microbiol Biotechnol 2018; 102: 1-7.

13. Zojaji H, Ghobakhlou M, Rajabalinia H. The efficacy and safety of adding the probiotic Saccharomyces boulardiito standard triple therapy for eradication of $\mathrm{H}$. pylori: a randomized controlled trial. Gastroenterol Hepatol 2013; 6: 99-104.

14. Sheu BS, Wu JJ, Lo CY. Impact of supplement with Lactobacillus and Bifidobacterium-containing yogurt on 
triple therapy for Helicobacter pylori eradication. Alimentary Pharmacol Therap 2002; 16: 1669-1675.

15. Canducci F, Armuzzi A, Cremonini F. A lyophilized and inactivated culture of Lactobacillus acidophilus increases Helicobacter pylori eradication rates. Alimentary Pharmacol Therap 2000; 14: 1625-1629.

16. Yang YJ, Chuang CC, Yang HB, Lu CC, Sheu BS. Lactobacillus acidophilus ameliorates $\mathrm{H}$. pylori-induced gastric inflammation by inactivating the Smad7 and NF kappa B pathways. BMC Microbiol 2012; 12: 38.

\section{"Correspondence to}

Lili Jiang

Department of Gastroenterology

General Hospital of Xinjiang Military Region

Urumqi

PR China 